\title{
Working with Daily Climate Model Output Data in $R$ and the futureheatwaves Package
}

by G. Brooke Anderson, Colin Eason, and Elizabeth A. Barnes

\begin{abstract}
Research on climate change impacts can require extensive processing of climate model output, especially when using ensemble techniques to incorporate output from multiple climate models and multiple simulations of each model. This processing can be particularly extensive when identifying and characterizing multi-day extreme events like heat waves and frost day spells, as these must be processed from model output with daily time steps. Further, climate model output is in a format and follows standards that may be unfamiliar to most R users. Here, we provide an overview of working with daily climate model output data in $\mathrm{R}$. We then present the futureheatwaves package, which we developed to ease the process of identifying, characterizing, and exploring multi-day extreme events in climate model output. This package can input a directory of climate model output files, identify all extreme events using customizable event definitions, and summarize the output using user-specified functions.
\end{abstract}

\section{Introduction}

Research on climate change impacts can require extensive processing of climate model output data. This is not only because output files for a single climate model can be large, but also because of the rising popularity of ensemble techniques (Cubasch et al., 2013) in which, to better characterize uncertainty in projections, impacts are assessed for multiple climate models, multiple simulations of each climate model, and multiple climate experiments. Such ensemble techniques help characterize uncertainty in projections of regional climate change over the next century due to three distinct sources: (1) internal climate variability, i.e., climate noise, (2) climate model uncertainty, i.e. the same forcing can produce a different response in different models and (3) scenario uncertainty, i.e., uncertainty in future climate forcings (e.g. Hawkins and Sutton (2009)).

A key source of data for ensemble techniques is the Coupled Model Intercomparison Project, Phase 5 (CMIP5; Taylor et al. (2012)). This project brought together major climate modeling groups around the world to simulate the same future radiative forcing scenarios, but with their own models. This created an ensemble of state-of-the-art climate model projections that allows researchers to study projections and their uncertainties. Most of these modeling groups additionally performed more than one simulation for each scenario and model (i.e. multiple ensemble members), perturbing the initial conditions by a very tiny amount to quantify uncertainties due to internal climate variability.

We begin this article with an overview of CMIP5 climate model output data for R users, focusing on output with a daily time step. We outline where data from CMIP5 can be obtained as well as how to work with the file format (netCDF) from R. We overview some R packages that can be useful when working with this data, as well as aspects of the data (e.g., non-standard calendars) of which users should be aware when working with daily climate model output in R.

After this overview, we present the futureheatwaves package, which we created to aid in identifying and characterizing any type of multi-day extreme event from daily climate model output (Table 1). The impacts of multi-day extreme events must be assessed using output in daily time step, unlike other climate impacts that can be assessed using climate model output at monthly, seasonal, or yearly time steps. Further, extreme events are identified based on conditions that are rare for a certain location (e.g., 98th percentile of local temperature distribution for identifying heat waves) (Cubasch et al., 2013). In this case, the event definition must be determined at each study location from climate model output before events can be identified. Finally, it is often of interest to create summaries of multiple characteristics of these extreme events. For example, one may be interested in determining whether the frequency or characteristics (e.g., length, intensity) of heat waves or warm spells will change under certain climate change scenarios (Cubasch et al., 2013).

The futureheatwaves package handles these challenges and can be used to identify and characterize a variety of multi-day extreme events across different ensemble members of one or more climate models. It also provides some functionality specifically useful in identifying and characterizing heat waves. Quantification of the impacts of heat waves on human health suffers from additional sources of uncertainty beyond those inherent in projections of regional changes in surface temperature. These include: (1) uncertainty in the definition of a heat wave itself and (2) uncertainty in the ability of communities to adapt to changing temperatures (the adaptation scenario). This package therefore 


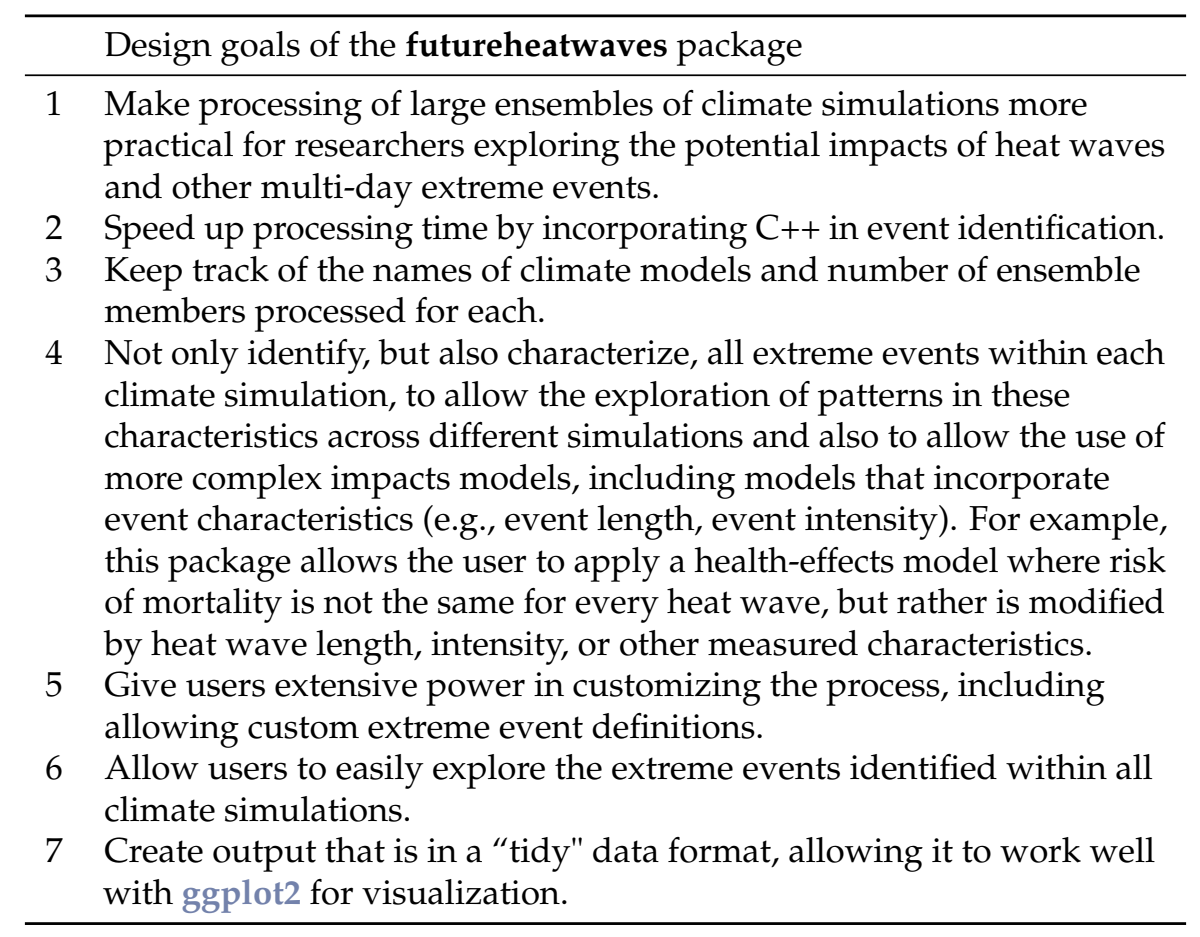

Table 1: Design goals for the futureheatwaves package.

allows the user to create and use a custom extreme event definition to identify events in the climate model output, as well as providing options to explore different scenarios of adaptation to heat.

\section{An overview of climate model output for $\mathbf{R}$ users}

\section{CMIP5 climate model output data}

For climate impact studies, a main source of data is the Coupled Model Intercomparison Project, which is currently in its fifth phase (CMIP5). Over 20 climate modeling groups created one or more climate models which, for this project, were run using standardized scenarios (Taylor et al., 2012). The resulting output is uniform across modeling groups and has a consistent structure, which allows comparison of simulations from different models (Flato et al., 2013). CMIP5 climate model output is archived at a number of different time steps (e.g., daily, monthly, seasonal, yearly) (Taylor and Doutriaux, 2010), and some variables are reported at multiple levels in the ocean or atmosphere (e.g., ocean temperature is reported at different ocean depths). Here, we will focus on data with a daily time step for variables reported at a single level (e.g., near-surface air temperature).

Each modeling group ran simulations for CMIP5 under several experiments, with experiments varying in terms of radiative forcing scenarios through the use of different scenarios of time-varying model inputs (greenhouse gas emissions or concentrations, land use changes, etc.) (Taylor et al., 2012; Flato et al., 2013). Experiments include historical experiments (run using radiative forcing consistent with observed and reconstructed data for 1850-2005), pre-industrial control experiments, and experiments of future scenarios of radiative forcing over the 21st century or longer (e.g., RCP4.5, RCP8.5) (Taylor et al., 2012). Some modeling groups created ensembles of output for a specific model and experiment, in which they ran the experiment multiple times with very small changes to the initial conditions.

The CMIP5 climate model output data are distributed across data nodes at different climate modeling centers (Taylor et al., 2012), but can be accessed centrally at the World Climate Research Programme CMIP5 data portal at https://pcmdi.1lnl.gov/search/cmip5/. Users must register before downloading data, and some data are restricted to non-commercial use. There is a separate file for each combination of climate model, experiment, modeling realm (e.g., atmosphere, ocean), variable, time step, and ensemble member (Taylor et al., 2012; Taylor and Doutriaux, 2010). For finer time scales, the output is further split across multiple files for specific year ranges (e.g., 5 years of output for each file) (Taylor and Doutriaux, 2010). Each file's name includes the output variable, climate model, experiment, and ensemble member for the simulation (Taylor and Doutriaux, 2010). 

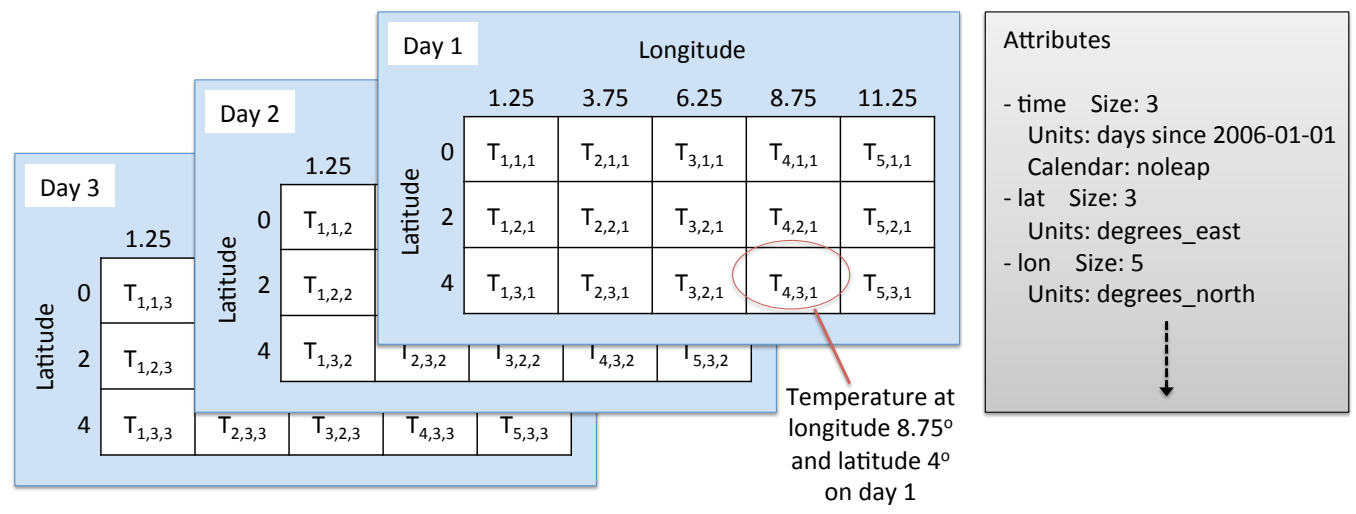

Figure 1: Example structure of a netCDF climate model output file for a variable reported at a single level, like near-surface air temperature. Data are stored in a three-dimensional array, with measurements at each time step and climate grid location. These data are typically indexed in the netCDF file by longitude, latitude, and time, in that order. For example, if the near-surface air temperature in the example netCDF shown here is read into an $\mathrm{R}$ object called tas, you can access the value for the first day at the fourth longitude and third latitude with $\operatorname{tas}[4,3,1]$. In addition to the output variable (temperature in this example), vectors with the ordered values of each dimension (longitude, latitude, and time) can also be read in from the netCDF file, as well as attribute data (e.g., units for variables, the calendar used for time).

CMIP5 files can be searched and downloaded through a point-and-click web interface. They can also be downloaded in bulk to computers with Unix or Mac operating systems using the wget file downloading utility. Appropriate wget scripts can be created either through the World Climate Research Programme CMIP5 data portal or through the Earth System Grid Federation's Search RESTful API. Tips on efficiently searching and downloading the data, including through the use of wget scripts and the search API, are available as user tutorials through the website of the University of Colorado Boulder's Earth System CoG (e.g., https: //www. earthsystemcog.org/projects/cog/doc/wget for a tutorial on downloading files using wget).

CMIP5 files are saved in Network Common Data Format (netCDF), a binary file format that allows storage of data representing a regular array. For climate model output at a single level (e.g., nearsurface air temperature), the data is a 3-dimensional array, with dimensions representing time and two coordinates of location (e.g., latitude and longitude). Figure 1 provides a sketch of the structure of netCDF files for single-level climate model output.

Each data point in the netCDF array gives the modeled value of the variable (e.g., surface temperature) for a single time point and location. Global climate models generate output at regularly-spaced time steps, typically at regularly-spaced grid points around the world. The latitude and longitude spacing of grid points vary by climate model, but are typically 1-2 degrees for atmospheric variables in CMIP5 models (Flato et al., 2013). For CMIP5 climate model output, the location units are in degrees east and degrees north for longitude and latitude, respectively. For daily output files, the time unit is in days since a specified origin date-time (e.g., days since 1850-01-01 00:00:00) (Taylor and Doutriaux, 2010).

All CMIP5 output files are required to include certain metadata (or "attributes") (Taylor and Doutriaux, 2010), including the experiment, forcing agents input to the model to create the simulation, time step, institution and institutional contact information, climate model, and modeling realm (Taylor and Doutriaux, 2010). The metadata also must include units for all of the dimension variables (e.g., longitude, latitude, time). The netCDF format allows one to access metadata and variables describing the dimensions of the data without reading the full file into memory.

To find out more about the CMIP climate model output data, Taylor et al. (2012) and Meehl et al. (2007) are excellent resources.

\section{Working with climate model output in $\mathbf{R}$}

When working with daily climate model output data, challenges to R users include: (1) the file format, (2) use of non-Gregorian calendars, and (3) large file sizes. This section explains these challenges and offers some strategies for dealing with them.

CMIP5 data are available in the netCDF file format. Free specialty software exists to work with 


\section{Modeled temperature on a day in July 2075 \\ GFDL-ESM2G model, RCP8.5 experiment, r1i1p1 ensemble member}
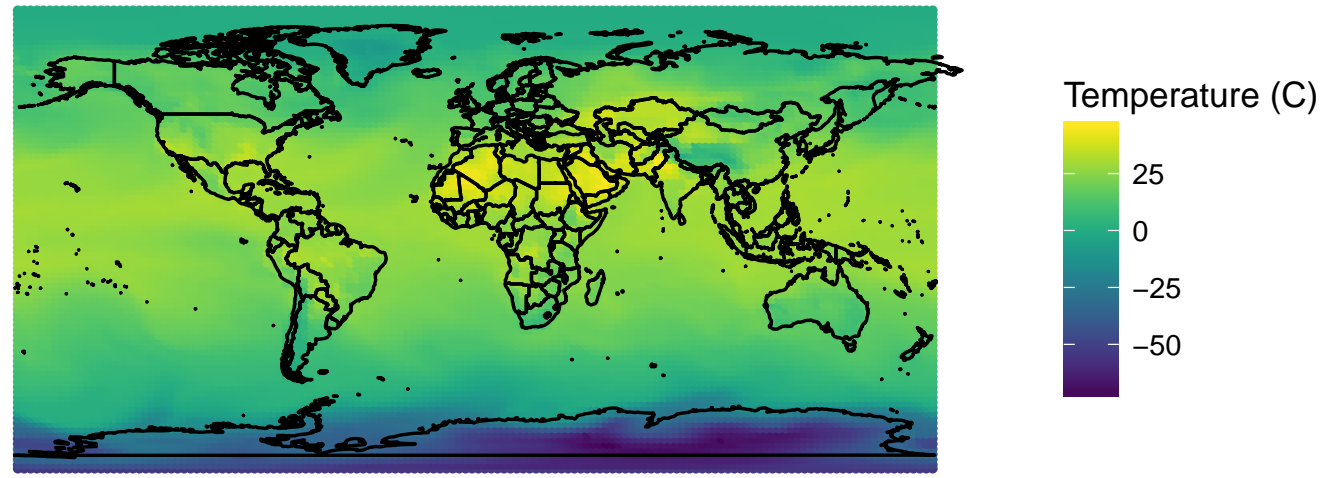

Figure 2: Example of mapping near-surface air temperature data worldwide for a single day of climate model output data. This map uses data from the Geophysical Fluid Dynamics Laboratory's Earth System Model 2G, r1i1p1 ensemble member, on a single day in the summer of 2075. Full code for recreating the map is available in the "starting_from_netcdf" vignette of the futureheatwaves package.

climate model output files in this format, including a collection of command line tools developed by the Max Planck Institute called Climate Model Operators (CDO) (Schulzweida, 2017) and an interpreted language developed by the National Center for Atmospheric Research (NCAR) called the NCAR Command Language (NCL) (UCAR/NCAR Computational and Information Systems Laboratory, 2017). Although such software can be used to quickly process netCDF climate model output files, they require learning a new language syntax, and so for $\mathrm{R}$ users may not be worth the computational speed gain compared to alternative solutions that can be scripted in the $\mathrm{R}$ language. While base $\mathrm{R}$ import functions do not exist for netCDF files, there are a few $\mathrm{R}$ package extensions that allow $\mathrm{R}$ users to work with the netCDF file format used for CMIP5 files directly from R. Older packages include ncdf and ncvar, but these do not work with the newer netCDF version 4 released in 2008 and are no longer available through CRAN. More recent packages, including ncdf4 (Pierce, 2015) and RNetCDF (Michna and Woods, 2013, 2016), work with both version 4 and netCDF's older version 3. While climate model output data for CMIP5 are required to conform with the earlier version (version 3) (Taylor and Doutriaux, 2010), it is safer to write code using functions that can be used with either version, in case future phases of CMIP do not require files to conform with netCDF version 3.

You can do a number of things with netCDF files in R using these packages. For example, ncdf4's nc_open function can be used to open a connection to a netCDF file; the object returned by the function includes the file's attribute data. Once a file connection is open, variables can be read in using the ncvar_get function. For example, the variables defining the dimensions of the sketched netCDF file in Figure 1 could be read into R with ncvar_get with the varid parameter set to "lat", "lon", or "time".

The climate output variable (e.g., near-surface air temperature) can similarly be read in using ncvar_get. In this case, the varid parameter should be set using the appropriate CMIP5 variable name (e.g., "tas" for near-surface air temperature); these variable names can be found in the CMIP requested output tables (Taylor and Doutriaux, 2010). If only a subset of the full file is needed, the dimensional time and location data can be used to identify the location of the needed data in the netCDF array and this information can then be used to read a portion of data into memory (e.g., with the nc.get.var. subset. by. axes function in ncdf4.helpers). Once the user is done reading in data from the file, the connection to the netCDF should be closed (e.g., with the nc_close function from ncdf4).

A second challenge when working with climate model output data in $\mathrm{R}$ is that some climate models output to non-Gregorian calendars. Since the late 1500s, Western dates have been set using the Gregorian calendar, which has 365.2425-day years. Some climate models, however, are run using different calendars, including the Julian calendar (365.25-day years), a calendar where there are no leap years (365-day years), a calendar where every year is a leap year (366-day years), and a calendar of twelve 30-day months (360-day years) (Eaton et al., 2011). With these non-Gregorian calendars, R's base functions for converting a vector to a Date class based on the number of days since an origin date (as.Date, as.POSIXct) do not return the desired values. 
Daily modeled near-surface air temprature, 2071-2075

At model grid point nearest Beijing, China

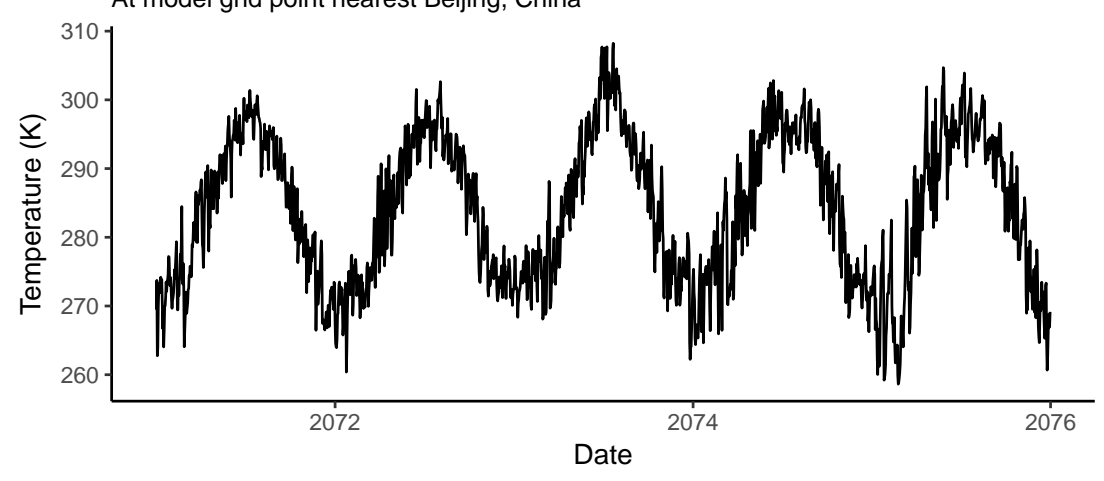

Figure 3: Example of plotting a time series of temperature simulations between 2071 and 2075 from CMIP5 daily climate model output data for the model grid cell point closest to Beijing, China. This plot uses data from the Geophysical Fluid Dynamics Laboratory's Earth System Model 2G, r1i1p1 ensemble member. Full code for recreating the map is available in the "starting_from_netcdf" vignette of the futureheatwaves package.

Two R packages provide help with non-Gregorian calendars: PCICt (Bronaugh and Drepper, 2013) and ncdf4.helpers (Bronaugh, 2014). The nc.get.time. series function in ncdf4.helpers pulls and uses metadata on the calendar stored in the CMIP5 netCDF file's attributes to convert the "time" variable in the file to an object of the PCICt class. This class is defined in the PCICt package and provides date-like functionality for 360- and 365-day calendars (Bronaugh and Drepper, 2013). However, while these functions will help with handling most CMIP5 files, the CMIP5 standards allows use of other calendars which may not be successfully handled by these functions, so it is important to assess whether the time variable range in the PCICt object correctly matches the expected date ranges for a file when processing CMIP5 data in R. While most CMIP5 climate models use the same calendar for all experiments, a few do not; a full table of the calendars used for each climate model and experiment, pulled from netCDF metadata, is available at https://www. earthsystemcog.org/ projects/cog/faq_data.

Finally, the size of CMIP5 files can make them difficult to work with in R. CMIP5 climate model output files can be as large as several gigabytes. The size of the files can therefore be large enough that it may make more sense to work with smaller chunks of the data in $\mathrm{R}$, rather than reading all data into memory and working with the data all at once (Todd-Brown and Bond-Lamberty, 2016). This problem aggregates when working with multiple climate models and more than one ensemble member for each of those climate models.

In addition to these general packages for working with netCDF files, there are several $\mathrm{R}$ packages specifically for working with climate model output data, including RCMIP5 (Todd-Brown and BondLamberty, 2016) and wux (Mendlik et al., 2016). However, these packages are more useful for working with data output at time steps of a month or higher and have limited utility with the daily climate model output data required for studies of multi-day extreme events.

The RCMIP5 package includes functions to read in CMIP5 data from netCDF files, scan a directory of CMIP5 files and determine models with continuous available data, create objects of a special cmip5data class to work with CMIP5 data within R, and parse the file names for all files in a directory to extract information within the file name. For this package, most functions only work with monthly or less frequent data (Todd-Brown and Bond-Lamberty, 2016). While the loadCMIP5 function does successfully load daily data as a cmip5data object, most of the methods for this object type do not do anything meaningful for daily data. The package's getFileInfo function, however, will work with CMIP5 files of any time step; this function identifies all CMIP5 files in a directory and creates a data frame with information parsed from the file name. The get.split.filename.cmip5 function in the ncdf4.helper package similarly can be used to parse information contained in CMIP5 file names (Bronaugh, 2014).

The wux package (Mendlik et al., 2016) includes functions that allow the user to download CMIP5 output at a monthly time step directly from $\mathrm{R}$ with the CMIP5fromESGF function. The package then uses the models2wux function to read climate model output netCDF files and convert it to "WUX" data frames, which can be used by other functions in the package. While this function can input climate model output with daily time steps (the "what.timesteps" element of the modelinput list input must be set to "daily"), the function aggregates this data to a monthly or less frequent (e.g., seasonal) 


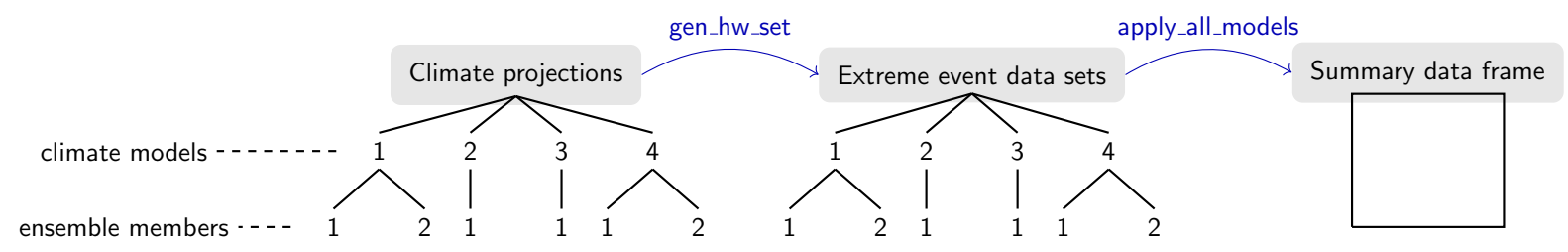

Figure 4: Overview of the functionality of the futureheatwaves package. The package takes a directory with climate projection files (left), for one or more climate models, with one or more ensemble members for each climate model (this example figure shows four climate models with one or two ensemble members each). The gen_hw_set function processes these files to create a data frame for each ensemble member, identifying and characterizing all multi-day extreme events (e.g., heat waves) in the time series projection for that ensemble member. The apply_all_models function allows users to explore these extreme events by applying user-created functions across all the extreme event data frames, creating a summary data frame with results.

aggregation when creating the WUX data frame. Therefore, while this package provides very useful functionality for working with averaged output of daily climate model output data or with output data at a larger time step, it cannot easily be used to identify and characterize multi-day extreme events like heat waves.

The functions and packages described in this section can be used with CMIP5 netCDF files to do things in R like map near-surface air temperatures from a single climate model on a specific day (Figure 2) or pull a time series of daily near-surface air temperature simulations at a specific climate model grid point (Figure 3). The futureheatwaves" "Starting from netCDF files" vignette (https://cran . r-project.org/web/packages/futureheatwaves/vignettes/starting_from_netcdf.html) provides all code required to create these figures, as well as more details and code examples on working with CMIP5 netCDF files in $\mathrm{R}$.

\section{The futureheatwaves package}

\section{Motivation}

We created the futureheatwaves package to aid in identifying, characterizing, and exploring multiday extreme events in daily climate model output data. While most of the discrete tasks involved in identifying and characterizing multi-day extreme events are fairly straightforward, the full process can be code-intensive, especially for multi-city studies, studies that test sensitivity to how an event is defined, or studies that incorporate different scenarios of adaptation in the case of events defined using a threshold relative to community climate. Our aim in developing this package was therefore to make the full process of identifying and characterizing these extreme events much more convenient and so facilitate the use of multi-model, multi-ensemble member analyses in climate impact studies conducted by non-climate scientists.

\section{How the package works}

Figure 4 gives an overview of the two primary functions of the futureheatwaves package. First, the gen_hw_set function processes a directory of climate projection files that are stored locally on the user's computer (Figure 4, "Climate projections"), to generate a list of all extreme events in each projection, as well as over a dozen characteristics of each identified extreme event (Table 2). This package start from files rather than $\mathrm{R}$ objects to avoid loading data from all climate model ensembles at once; instead, the function loads, processes, and saves output for a single climate model ensemble member at a time. The extreme events are identified and characterized at one or more study locations (e.g., cities), which the user specifies in an input file. The extreme events identified for each ensemble member are output as separate files in a directory specified by the user (Figure 4, "Extreme events datasets").

Once the user creates these data frames of location-specific extreme events, the apply_all_models function can be used to apply custom functions across all the extreme event data frames. This functionality allows users to create summaries of extreme events across all climate models and ensemble members (Figure 4, right). The function can be used to generate summary statistics (e.g., determine average heat wave length or total frost days) or to apply more complex functions (e.g., apply epidemiologic effect estimates across the heat waves to generate health impact estimates). 
When using this package, CMIP5 climate model output data require some pre-processing. Data will need to be saved in a specific format, with files stored in a specific directory structure. Full details of the required file and directory structure are provided in the package's main vignette (https: //cran.r-project.org/web/packages/futureheatwaves/vignettes/futureheatwaves.html), while tips and an R script for conducting this processing starting from CMIP5 netCDF files are given in the "Starting from netCDF files" vignette.

This package can be used for study locations worldwide. The "Starting from netCDF files" package vignette provides an example of using this package to identify and explore future heat waves in several Chinese cities.

\section{Example data}

We have included data files in the package to serve as example files so that users can try this package before applying it to their own directory of climate projection files. These example data come from two climate models that are a part of CMIP5: (1) the model of the Beijing Climate Center, China Meteorological Administration (BCC) (Xin et al., 2013) and (2) the National Center for Atmospheric Research's (NCAR's) Community Climate System Model, version 4 (CCSM4) (Gent et al., 2011). We include one ensemble member from BCC (r1i1p1) and two from CCSM (r1i1p1 and r2i1p1). Once the futureheatwaves package is installed and loaded, the user can find the location of these files on his or her computer using R's system. file function.

To ensure that the size of this example data is reasonably small, we have only included projection data for grid points from these climate models that are near five U.S. east coast cities: New York, NY; Philadelphia, PA; Newark, NJ; Baltimore, MD; and Providence, RI. Further, to keep the file sizes reasonably small, the historical projections range over the years 1990 to 1999, while the future projections are limited to 2060 to 2079. Users' applications of this package will likely use directories with many more climate model ensemble members and more locations; however, the operation of the package is the same for this smaller example application, as it would be for a much larger application.

\section{Basic example of using futureheatwaves}

Once climate model output files are set up, as specified in the "futureheatwaves" package vignette, the package can process them to identify and characterize heat waves in each ensemble member's projection for each location using the gen_hw_set function. For example, to process the example climate model output data included with the package, the user can run:

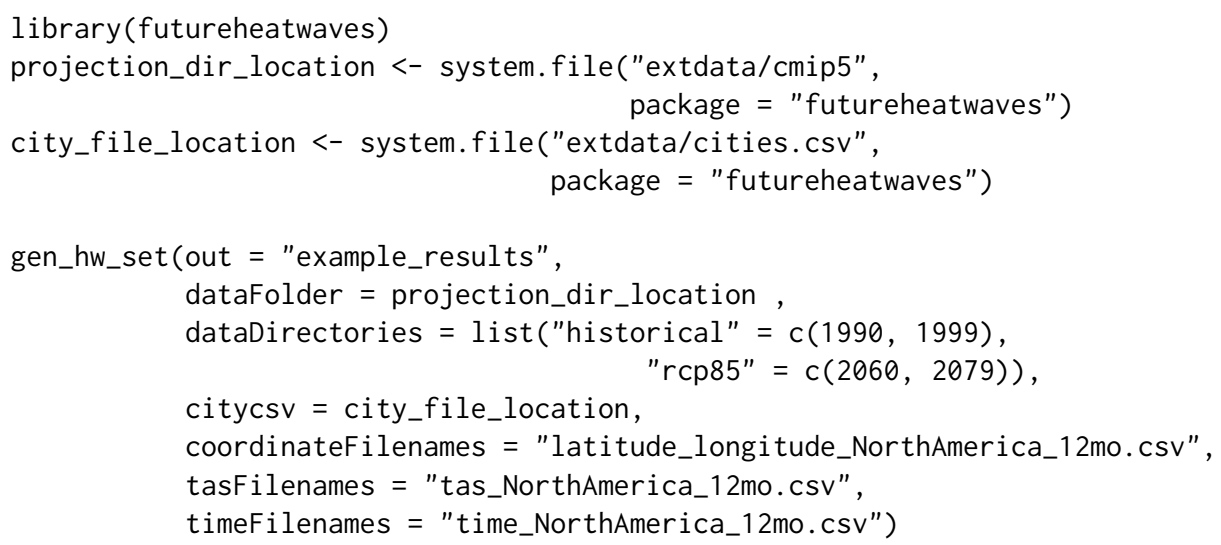

This code first identifies and saves as objects the path names on the user's computer of the example climate projections directory (projection_dir_location) and the file of study locations (city_file_location). The gen_hw_set function processes the example input and creates a new directory, 'example_results', with files of identified and characterized heat waves, in the user's current working directory. In this example code, the processing is done using default values for the event definition, adaptation scenario, etc. How and why to customize these choices are explained later in the text. Function arguments (e.g., dataDirectories, tasFilenames) are used to specify the format of the data and the directory structure.

Once the function has completed running, results will be written locally to the directory specified by the out argument of gen_hw_set. This directory will include files with some basic information about the climate models and the closest grid points of each climate model to each location, as well as a directory with files of identified and classified extreme events for each ensemble member, including 


\begin{tabular}{|c|c|}
\hline Column name & Description of characteristic \\
\hline mean.var & $\begin{array}{l}\text { Average daily value of the variable across all days in the extreme } \\
\text { event, in the units in which the variable is expressed in input files (e.g., } \\
\text { average daily mean temperature during the heat wave in degrees } \\
\text { Kelvin) }\end{array}$ \\
\hline $\max$. var & $\begin{array}{l}\text { Highest daily value of the variable across all days in the extreme event, } \\
\text { in the units in which the variable is expressed in input files }\end{array}$ \\
\hline min.var & $\begin{array}{l}\text { Lowest daily value of the variable across all days in the extreme event, } \\
\text { in the units in which the variable is expressed in input files }\end{array}$ \\
\hline length & Number of days in the event \\
\hline start.date & Date of the first day of the event \\
\hline end. date & Date of the last day of the event \\
\hline start.doy & Day of the year of the first day of the event $(1=$ Jan. 1 , etc. $)$ \\
\hline start.month & Month in which the event started ( $1=$ January) \\
\hline days.above.abs. thresh. 1 & $\begin{array}{l}\text { Number of days in the event above a specified absolute threshold } \\
\text { (default is the number of days in the event above } 80^{\circ} \mathrm{F} / 26.7^{\circ} \mathrm{C} \text {, but } \\
\text { this and the following three absolute thresholds can be changed with } \\
\text { the absolute_thresholds argument in gen_hw_set) }\end{array}$ \\
\hline days.above.abs. thresh. 2 & $\begin{array}{l}\text { Number of days in the event above a specified absolute threshold } \\
\text { (default is the number of days in the event above } 85^{\circ} \mathrm{F} / 29.4^{\circ} \mathrm{C} \text { ) }\end{array}$ \\
\hline days. above. abs. thresh. 3 & $\begin{array}{l}\text { Number of days in the event above a specified absolute threshold } \\
\text { (default is the number of days in the event above } 90^{\circ} \mathrm{F} / 32.3^{\circ} \mathrm{C} \text { ) }\end{array}$ \\
\hline days.above.abs. thresh. 4 & $\begin{array}{l}\text { Number of days in the event above a specified absolute threshold } \\
\text { (default is the number of days in the event above } 95^{\circ} \mathrm{F} / 35.0^{\circ} \mathrm{C} \text { ) }\end{array}$ \\
\hline days.above.99th & $\begin{array}{l}\text { Number of days in the event above the } 99^{\text {th }} \text { percentile of the variable } \\
\text { for the location, using the period specified with the } \\
\text { referenceBoundaries argument in gen_hw_set as a reference for } \\
\text { determining these percentiles }\end{array}$ \\
\hline days.above.99.5th & $\begin{array}{l}\text { Number of days in the event above the } 99.5^{\text {th }} \text { percentile of the variable } \\
\text { for the location, using the period specified with the } \\
\text { referenceBoundaries argument in gen_hw_set as a reference for } \\
\text { determining these percentiles }\end{array}$ \\
\hline first.in.year & $\begin{array}{l}\text { Whether the event was the first to occur in its calendar year in the } \\
\text { location }\end{array}$ \\
\hline mean.var.quantile & $\begin{array}{l}\text { The percentile of the average variable value during the event } \\
\text { compared to the location's year-round distribution of the variable, } \\
\text { based on the variable distribution for the location during the period } \\
\text { specified by the referenceBoundaries argument in gen_hw_set }\end{array}$ \\
\hline max.var.quantile & $\begin{array}{l}\text { The percentile of the maximum variable value during the event } \\
\text { compared to the location's year-round distribution of the variable, } \\
\text { based on the variable distribution for the location during the period } \\
\text { specified by the referenceBoundaries argument in gen_hw_set }\end{array}$ \\
\hline min.var.quantile & $\begin{array}{l}\text { The percentile of the minimum variable value during the event } \\
\text { compared to the location's year-round distribution of the variable, } \\
\text { based on the variable distribution for the location during the period } \\
\text { specified by the referenceBoundaries argument in gen_hw_set }\end{array}$ \\
\hline mean. seasonal.var & $\begin{array}{l}\text { The location's average seasonal value of the variable (by default, } \\
\text { season is set to May-September, but this can be changed with the } \\
\text { seasonal_months argument in gen_hw_set), based on the variable } \\
\text { values for the location during the years specified by the } \\
\text { referenceBoundaries argument in gen_hw_set }\end{array}$ \\
\hline mean. yearround. var & $\begin{array}{l}\text { The location's average year-round value of the variable, based on the } \\
\text { variable values for the location during the years specified by the } \\
\text { referenceBoundaries argument in gen_hw_set }\end{array}$ \\
\hline
\end{tabular}

Table 2: Extreme event characteristics measured by the gen_hw_set function in the futureheatwaves package. The left column gives the name of each variable's column in the extreme event data frames created by the gen_hw_set function. When characterizing extreme events below a threshold, like cold spells, appropriate alternatives are given for some columns (e.g., days.below.abs.thresh.1, days. below. 1st). 
all characteristics in Table 2. See the package's vignettes for more details on the content and structure of this output.

Once uses have created a directory of characterized event files for each ensemble member ("Extreme event data sets", Figure 4), they can explore the results using the apply_all_models function. This function allows the user to apply custom R functions across all extreme event data frames created by the gen_hw_sets call. The user can apply any $\mathrm{R}$ function that follows certain standards in accepting input and returning output. Full details on these standards are given in the main package vignette.

As an example, if the user wanted to calculate the average temperature of the heat waves identified for each ensemble member in the output generated by the code above, he or she could write a simple function:

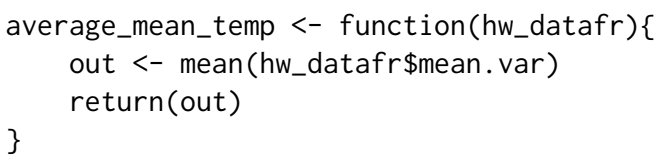

This function can then be applied across all extreme event data sets output by gen_hw_set using the apply_all_models function. For example, to apply this function to all the example output results that come with the package, the user could run:

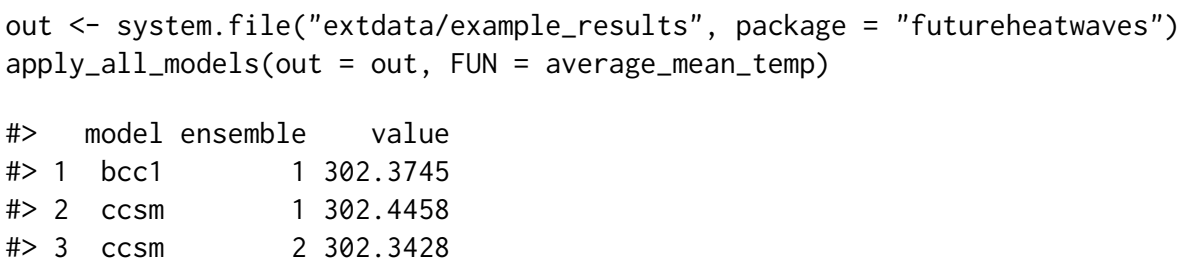

This output gives the results (value column) of running the custom function for each ensemble member of each climate model. Note that the location of the directory with the heat wave data frames must be specified using the out argument when calling apply_all_models. Typically, this will be the directory path for the directory specified with the out argument in gen_hw_set.

Location-specific results can be generated using the city_specific argument in apply_all_models:

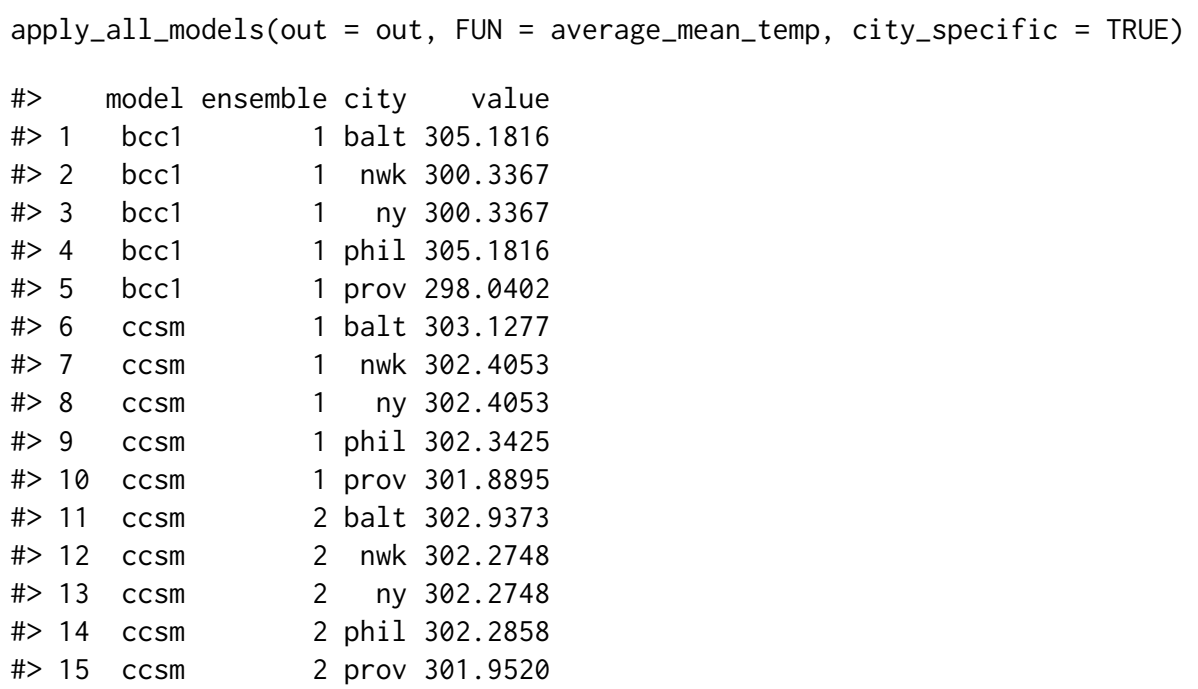

The same process can be used to create a number of other summaries of the identified extreme events. For example, it could be used to determine average length of extreme events or estimate how much earlier in the year events are expected to start across an ensemble of climate model simulations. The functionality can also be used for more complex analysis of extreme event files. For example, it can be used to apply epidemiological models of heat wave to estimate excess heat-related mortality under different future scenarios; an example of this application is provided in the main futureheatwaves vignette. The output from apply_all_models is structured as "tidy" data (Wickham, 2014), allowing it to be used easily with the graphing package ggplot2 (Wickham, 2009) and other packages in the tidyverse. 


\section{Customizing the extreme event definition}

By default, the package identifies extreme events in climate model output data using a specific definition for heat waves that has been used in some epidemiological and climate impact research (e.g., Anderson and Bell (2009)):

A heat wave is two or more days at or above a city-specific threshold temperature, with the threshold determined as the $98^{\text {th }}$ percentile of year-round temperature in the city during some reference period (by default, 1990-1999).

However, this is not the only accepted heat wave definition in the scientific literature. A variety of different heat wave definitions have been used to identify heat waves in a time series of temperature data (Smith et al., 2013; Kent et al., 2014; Chen et al., 2015; Anderson and Bell, 2009), and the choice of heat wave definitions can influence both projected heat wave trends (Smith et al., 2013) and estimates of health risks during events (Chen et al., 2015; Kent et al., 2014; Anderson and Bell, 2009). Further, other types of extreme events will be defined differently than heat waves (for example, frost day spells may be defined as one or more days with temperature at or below $32^{\circ} \mathrm{F} / 0^{\circ} \mathrm{C}$ ).

Therefore, this package allows the user to extensively customize the definition used to identify extreme events. Users can write a custom $R$ function with either a different heat wave definition (see Smith et al. (2013) and Kent et al. (2014) for listings of some of the definitions used in scientific studies) or with a definition appropriate for a different type of extreme event (e.g., one or more days at or below $32^{\circ} \mathrm{F} / 0^{\circ} \mathrm{C}$ for frost day spells). For heat wave identification, researchers might want to use a different event definition because, for example, it matches the definition used by local health officials to declare heat wave warnings or, in the case of health impact assessments, to match with a definition used in an epidemiological study. For studies of other extreme events, a heat wave definition likely will not be applicable and so a customized definition is necessary.

Three components of the extreme event definition can be easily customized in the gen_hw_set function call, without creating a new $\mathrm{R}$ function to use to identify heat waves. First, many extreme event definitions are based on conditions that are rare in the study location (Cubasch et al., 2013), but definitions may vary in how rare conditions must be. For example, some of the different definitions used to identify heat waves vary only in the percentile temperature used for a threshold (e.g., one definition is $\geq 2$ days at or above the $98^{\text {th }}$ percentile temperature at a location while another is $\geq 2$ days at or above the $99^{\text {th }}$ percentile temperature; Kent et al. (2014); Smith et al. (2013)). Therefore, the futureheatwaves package allows users to change the percentile of the variable of interest required for an extreme event using the probThreshold option in gen_hw_set. Other heat wave definitions vary only in the number of consecutive days that must be over the threshold for a period to quality as an extreme event (e.g., one definition is $\geq 2$ days at or above the $98^{\text {th }}$ percentile temperature at a location while another is $\geq 4$ days at or above the $98^{\text {th }}$ percentile temperature; Anderson and Bell (2009)). Therefore, the package allows the user to change the number of days used in the heat wave definition using the numDays argument in the gen_hw_set function. Combined, these two customization choices allow the user to identify heat waves using many of the heat wave definitions used in previous climate and health research-for example, 9 of 16 heat wave definitions outlined in Kent et al. (2014) could be fit using different combinations of these two options for specifying threshold percentile and number of days. Third, some extreme events like cold waves and frost day spells are defined as a certain number of days below, rather than above, a threshold. While the default is to identify events by searching for days above a threshold, this behavior can be changed with the above_threshold = FALSE argument in the gen_hw_set function.

Beyond these simpler options, the customization of the event definition is even more extensive as one has the option of writing and using a custom $\mathrm{R}$ function to identify extreme events. This functionality allows the user to use definitions that either require a number of days above or below an absolute threshold (e.g., maximum temperature of $\geq 95^{\circ} \mathrm{F}$ for $\geq 1$ day Kent et al. (2014); Tan et al. (2007); minimum temperature $\leq 0^{\circ} \mathrm{C}$ for $\geq 1$ day for frost day spells) or that require a combination of thresholds to be met (e.g., maximum daily temperature above a lower threshold every day of the heat wave and above a higher threshold for a certain number of days within the heat wave; Kent et al. (2014); Peng et al. (2011)). To use a customized event definition, the user must write and load an R function that implements the definition. This custom function is passed to the gen_hw_set function using the IDheatwavesFunction argument. To work correctly, this custom function must allow only specific inputs and generate only specific outputs; details about the required structure are provided in the main futureheatwaves package vignette. To increase processing speed when identifying extreme events, we coded parts of the default event definition function in $\mathrm{C}++$ and synced it with $\mathrm{R}$ using the Rcpp package (Eddelbuettel and Francois, 2011). Users should consider a similar strategy for custom heat wave definitions, especially when processing a large number of climate projection files. 


\section{Exploring sensitivity of results to adaptation}

Extreme events tend to be identified based on conditions that are rare for a specific location using location-specific relative thresholds. These thresholds are often defined for climate impact studies based on a variable's distribution at that location in present-day or historical data. However, for some extreme events, impacts are associated with how rare the conditions during the event are compared to current norms in the location (Anderson and Bell, 2009), which suggests some capacity for adaptation to heat and raises the question of whether extreme events should be defined using a percentile threshold based on present-day variable distributions or based on distributions in the time period being projected. Therefore, it can be interesting to explore trends in extreme events under climate change if extreme events are identified based on variable distributions during the projection period or another future period. The futureheatwaves package allows users to specify the time period to use when determining a location-specific relative threshold for an event definition using the thresholdBoundaries argument in the function gen_hw_set. This feature allows users to explore how sensitive projections of impacts are to this choice of the time period to use when determining relative variable measures, including thresholds used for percentile-based event definitions.

Similarly, some of the event characteristics (e.g., mean. temp. quantile, Table 2) are also calculated by the package based on relative temperature, providing measures of how the value of the variable of interest during an extreme event compares to the typical distribution of that variable at that location (e.g., the "mean.var.quantile", "min.var.quantile", and "max.var.quantile" characteristics, Table 2) or how long conditions of a certain rarity persisted during the event (e.g., the "days.above.99th" and "days.above.99.5th" characteristics, Table 2). These characteristics are measured for each of the extreme events identified by the gen_hw_set function by taking the absolute value of the variable during the event (e.g., average temperature during the heat wave is $90^{\circ} \mathrm{F}, 32.2^{\circ} \mathrm{C}$ ) and comparing it to the location's typical variable distribution. This process generates relative measures of how intense the event is compared to what is normal in that location (e.g., $90^{\circ} \mathrm{F}, 32.2^{\circ} \mathrm{C}$ is in the $99^{\text {th }}$ percentile of year-round temperatures in the location).

These relative event characteristics will vary depending on whether you calculate them based on a location's present-day variable distribution or on the location's variable distribution in the future, since the distributions of many relevant variables (e.g., temperature, precipitation) are expected to change in many locations with climate change. The package therefore allows the user to specify date ranges of the temperature distributions to be used in calculating these relative temperature metrics in each location, which can be done using the referenceBoundaries option of gen_hw_set.

\section{Mapping grid points}

Finally, it can be useful to explore the location of the climate model grid point used to pull climate model output for each study location with a given climate model. Therefore, the package has a function called map_grid_leaflet that plots the locations of grid points used for each location from each climate model. This function is built using the htmlWidget leaflet package (Cheng and Xie, 2016). The following code illustrates the use of this function with the example data to create Figure 5, which plots the grid points used in the example data from the BCC climate model in the example data:

out <- system.file("extdata/example_results", package = "futureheatwaves") map_grid_leaflet (plot_model = "bcc1", out = out)

This interactive map can be panned and zoomed to explore the locations of climate model grid points used to represent each study location. This mapping function works for study locations worldwide.

\section{Extensions}

While this package was created to be used for research on extreme events in climate change projections, it can be used more broadly. For example, there are other episodes like wildfires and air pollution where it may be interesting to identify extended periods of high exposures in projection time series. The futureheatwaves package is not exclusive to CMIP5 model output data, and so could be applied to gridded air pollution model output to explore these exposures.

\section{Future directions for working with climate model output in $\mathbf{R}$}

Research that assesses the potential impacts of climate change is critical in informing current policy choices, and $\mathrm{R}$ is an important tool for many researchers performing such assessments. While the futureheatwaves package described here takes steps to facilitate the assessment of impacts related to 


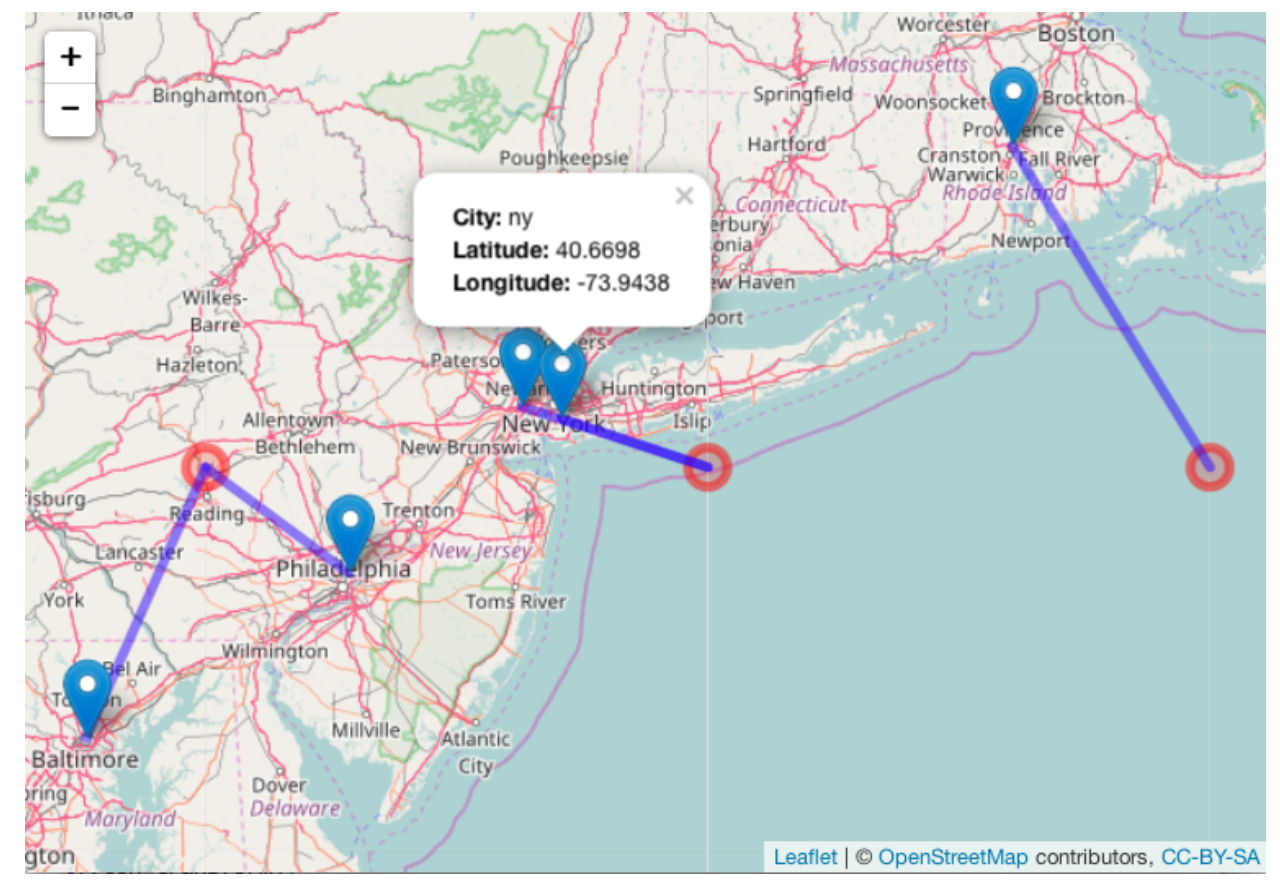

Figure 5: Snapshot of an interactive map created using the map_grid_leaflet showing the locations of study cities and their matching climate model grid points for the BCC climate model example data included with futureheatwaves. The lines on the map connect each climate model grid point to the study location(s) for which that grid point was used. The interactive maps include pop-ups with city identifiers; one is shown open in this snapshot as an example. From this map, you can see that the climate model grid point closest to New York City for this climate model is over the Atlantic Ocean.

sustained, multi-day events, a number of challenges remain in working with climate model output data in $\mathrm{R}$, and future $\mathrm{R}$ software development offers the potential to further address the challenges of working with this data.

One important step in future development of R software to work with climate model output could be the development of $\mathrm{R}$ wrappers for some of the existing command line tools available through the Climate Data Operators (CDO) software (Schulzweida, 2017). Libraries already exist for Python and Ruby that allow the functionality of $\mathrm{CDO}$ tools to be used within these scripting languages (available from the Max Planck Institute at https://code. zmaw. de/projects/cdo/wiki/Cdo\%7Brbpy\%7D). While one R package (ncdf4.helpers; Bronaugh (2014)) already provides R wrappers for a few CDO operators, such functionality could be extended through future $\mathrm{R}$ software to capture more of the full functionality of the CDO toolkit.

Another important path for development could be through approaches that allow researchers to take advantage of the statistical tools offered by $\mathrm{R}$ while maintaining large climate model output files in a netCDF format. For example, Goncalves and coauthors recently described a "round table" approach of connecting as-needed data access from netCDF climate data files through to functionality available in $\mathrm{R}$ and CDO through the intermediary of a MonetDB database system (Goncalves et al., 2015). In other topical areas, $R$ programmers are also improving the efficiency of working with data in large netCDF files through approaches that avoid loading all data in-memory. For example, the Bioconductor package ncdfFlow enables R users to conduct analyses of hundreds of large flow cytometry output files through the creation and use of an ncdfFlowset class that stores the data in an HDF5 format rather than in-memory (Jiang et al., 2017; Finak et al., 2014). Such an approach could be promising for future R software development for working with large climate model files.

\section{Acknowledgements}

This work was supported by grants from the National Institute of Environmental Health Sciences (R00ES022631), the National Science Foundation (1331399), and the Colorado State University Vice President for Research. Thank you to early testers of the package: Julia Bromberek, Wande BenkaCoker, Josh Ferreri, Ryan Gan, Molly Gutilla, Mike Lyons, Casey Quinn, Rachel Severson, and Meilin Yan. 


\section{Bibliography}

G. B. Anderson and M. L. Bell. Weather-related mortality: How heat, cold, and heat waves affect mortality in the United States. Epidemiology, 20(2):205-213, 2009. URL https: //doi . org/10. 1097/ ede.0b013e318190ee08. [p133,134]

D. Bronaugh. Ncdf4.helpers: Helper Functions for Use with the Ncdf4 Package, 2014. URL https: //CRAN . Rproject. org/package=ncdf 4. helpers. R package version 0.3.3. [p128, 135]

D. Bronaugh and U. Drepper. PCICt: Implementation of POSIXct Work-Alike for 365 and 360 Day Calendars., 2013. URL https://CRAN. R-project. org/package=PCICt. R package version 0.5.4. [p128]

K. Chen, J. Bi, J. Chen, X. Chen, L. Huang, and L. Zhou. Influence of heat wave definitions to the added effect of heat waves on daily mortality in Nanjing, China. Science of the Total Environment, 506-507:18-25, 2015. URL https://doi.org/10.1016/j.scitotenv.2014.10.092. [p133]

J. Cheng and Y. Xie. Leaflet: Create Interactive Web Maps with the JavaScript 'Leaflet' Library, 2016. URL https://CRAN.R-project.org/package=leaflet. R package version 1.0.1. [p134]

U. Cubasch, D. Wuebbles, D. Chen, M. C. Facchini, D. Frame, N. Mahowald, and J.-G. Winther. Introduction. In: Climate Change 2013: The Physical Science Basis. Contribution of Working Group I to the Fifth Assessment Report of the Intergovernmental Panel on Climate Change. Climate Change 2013, 5:119-158, 2013. [p124, 133]

B. Eaton, J. Gregory, B. Drach, K. Taylor, S. Hankin, J. Caron, R. Signell, P. Bentley, G. Rappa, H. Höck, and others. NetCDF Climate and Forecast (CF) Metadata Conventions Version 1.6, 2011. URL http://cfconventions.org/Data/cf-conventions/cf-conventions-1.6/build/cfconventions.html. [p127]

D. Eddelbuettel and R. Francois. Rcpp: Seamless R and C++ integration. Journal of Statistical Software, 40(8):1-18, 2011. URL https://doi.org/10.18637/jss.v040.i08. [p133]

G. Finak, J. Frelinger, W. Jiang, E. W. Newell, J. Ramey, M. M. Davis, S. A. Kalams, S. C. De Rosa, and R. Gottardo. Opencyto: An open source infrastructure for scalable, robust, reproducible, and automated, end-to-end flow cytometry data analysis. PLoS Comput Biol, 10(8):e1003806, 2014. [p135]

G. Flato, J. Marotzke, B. Abiodun, P. Braconnot, S. C. Chou, W. J. Collins, P. Cox, F. Driouech, S. Emori, V. Eyring, and others. Evaluation of Climate Models. In: Climate Change 2013: The Physical Science Basis. Contribution of Working Group I to the Fifth Assessment Report of the Intergovernmental Panel on Climate Change. Climate Change 2013, 5:741-866, 2013. [p125, 126]

P. R. Gent, G. Danabasoglu, L. J. Donner, and others. The Community Climate System Model Version 4. Journal of Climate, 24(19):4973-4991, 2011. URL https: //doi . org/10.1175/2011 jcli4083.1. [p130]

R. Goncalves, M. Ivanova, F. Alvanaki, J. Maassen, K. Kyzirakos, O. Martinez-Rubi, and H. Mühleisen. A round table for multi-disciplinary research on geospatial and climate data. In e-Science (e-Science), 2015 IEEE 11th International Conference on, pages 165-170. IEEE, 2015. [p135]

E. Hawkins and R. Sutton. The potential to narrow uncertainty in regional climate predictions. Bulletin of the American Meteorological Society, 90(8):1095-1107, 2009. URL https://doi.org/10. 1175/2009bams2607.1. [p124]

M. Jiang, G. Finak, and N. Gopalakrishnan. ncdfFlow: A Package That Provides HDF5 Based Storage for Flow Cytometry Data, 2017. R package version 2.22.0. [p135]

S. T. Kent, L. A. McClure, B. F. Zaitchik, T. T. Smith, and J. M. Gohlke. Heat waves and health outcomes in Alabama (USA): The importance of heat wave definition. Environmental Health Perspectives, 122 (2):151-158, 2014. URL https://doi .org/10.1289/ehp. 1307262. [p133]

G. A. Meehl, C. Covey, T. Delworth, M. Latif, B. McAvaney, J. F. B. Mitchell, R. J. Stouffer, and K. E. Taylor. The WCRP CMIP3 Multimodel Dataset: A new era in climate change research. Bulletin of the American Meteorological Society, pages 1383-1394, 2007. [p126]

T. Mendlik, G. Heinrich, and A. Leuprecht. Wux: Wegener Center Climate Uncertainty Explorer, 2016. URL https://CRAN. R-project.org/package=wux. R package version 2.2.1. [p128]

P. Michna and M. Woods. RNetCDF-a package for reading and writing netcdf datasets. The R Journal, 5:29-36, 2013. [p127] 
P. Michna and M. Woods. RNetCDF: Interface to NetCDF Datasets, 2016. URL https://CRAN.Rproject. org/package=RNetCDF. R package version 1.8.2. [p127]

R. D. Peng, J. F. Bobb, C. Tebaldi, L. McDaniel, M. L. Bell, and F. Dominici. Toward a quantitative estimate of future heat wave mortality under global climate change. Environmental Health Perspectives, 119(5):701-706, 2011. URL https://doi .org/10.1289/ehp. 1002430. [p133]

D. Pierce. Ncdf4: Interface to Unidata netCDF (Version 4 or Earlier) Format Data Files, 2015. URL https://CRAN.R-project. org/package=ncdf4. R package version 1.15.0. [p127]

U. Schulzweida. CDO User's Guide, 2017. URL https://code.zmaw. de/projects/cdo/embedded/cdo. pdf. Climate Data Operators, Version 1.8.1. [p127, 135]

T. T. Smith, B. F. Zaitchik, and J. M. Gohlke. Heat waves in the United States: Definitions, patterns and trends. Climatic Change, 118(3-4):811-825, 2013. URL https://doi .org/10. 1007/s10584-012-06592. [p133]

J. Tan, Y. Zheng, G. Song, L. S. Kalkstein, A. J. Kalkstein, and X. Tang. Heat wave impacts on mortality in Shanghai, 1998 and 2003. International Journal of Biometeorology, 51(3):193-200, 2007. URL https://doi.org/10.1007/s00484-006-0058-3. [p133]

K. E. Taylor and C. Doutriaux. CMIP5 Model Output Requirements: File Contents and Format, Data Structure and Metadata, 2010. URL http://cmip-pcmdi.1lnl.gov/cmip5/docs/CMIP5_output_metadata_ requirements.pdf. [p125, 126, 127]

K. E. Taylor, R. J. Stouffer, and G. A. Meehl. An overview of CMIP5 and the experiment design. Bulletin of the American Meteorological Society, 93(4):485-498, 2012. URL https: //doi .org/10.1175/bams-d11-00094.1. [p124, 125, 126]

K. Todd-Brown and B. Bond-Lamberty. RCMIP5: Tools for Manipulating and Summarizing CMIP5 Data, 2016. URL https://cran.r-project.org/web/packages/RCMIP5/. R package version 1.2.0. [p128]

UCAR/NCAR Computational and Information Systems Laboratory. The NCAR Command Language (NCL), 2017. URL https://doi .org/10.5065/d6wd3xh5. Version 6.4.0. [p127]

H. Wickham. Ggplot2: Elegant Graphics for Data Analysis. Springer-Verlag, 2009. ISBN 978-0-387-98140-6. [p132]

H. Wickham. Tidy data. Journal of Statistical Software, 59(10):1-23, 2014. doi: 10.18637/jss.v059.i10. URL https: //www. jstatsoft.org/v059/i10. [p132]

X.-G. Xin, T.-W. Wu, and Z. Jie. Introduction of CMIP5 experiments carried out with the climate system models of Beijing Climate Center. Advances in Climate Change Research, 4(1):41-49, 2013. URL https://doi.org/10.3724/sp.j.1248.2013.041. [p130]

G. Brooke Anderson

Colorado State University

Department of Environmental \& Radiological Health Sciences

1681 Campus Delivery

Fort Collins, Colorado 80523

brooke. anderson@colostate. edu

Colin Eason

Colorado State University

Department of Computer Science

1873 Campus Delivery

Fort Collins, Colorado 80523

aimesce@gmail.com

Elizabeth A. Barnes

Colorado State University

Department of Atmospheric Science

1371 Campus Delivery

Fort Collins, CO 80523

eabarnes@atmos.colostate.edu 